\title{
Die Unterschenkelvenenthrombose - eine Erkrankung für Spezialisten
}

\author{
J. Herold; R. Bauersachs \\ Klinik für Gefäßmedizin - Angiologie, Gefäßzentrum, Klinikum Darmstadt GmbH, Darmstadt
}

\author{
Schlüsselwörter \\ Muskelvenenthrombose, isolierte Unter- \\ schenkelthrombose, Thromboembolie, distale \\ Thrombose.
}

\section{Zusammenfassung}

Für Patienten mit proximalen Thrombosen oder Lungenembolien ist die antikoagulatorische Behandlung klar definiert. Sind distale Venen thrombosiert gibt es in den aktuellen nationalen und internationalen Leitlinien widersprüchliche Empfehlungen, welche von therapeutischer Antikoagulation über 3 Monate oder länger, über intermediäre Antikoagulation über einen kürzeren Zeitraum bis hin zum Verzicht auf Antikoagulation bei klinischer Überwachung reichen. Dieser Artikel soll hierüber anhand der Zusammenstellung aktueller Studien, Leitlinien und Expertenmeinungen die doch meist patientenindividuelle Therapieempfehlung kritisch beleuchten und dann erleichtern.

\section{Keywords}

Isolated calf vein thrombosis, isolated calf muscle vein thrombosis, thromboembolism, deep vein thrombosis.

\section{Summary}

For patients with proximal deep vein thrombosis or pulmonary embolism, the initial treatment is well defined. However, there are conflicting recommendations from current guidelines when distal (calf) veins are thrombosed, ranging from surveillance without anticoagulation to full therapeutic anticoagulation for three months or even longer, as the dose and duration of the anticoagulant is not yet established due to sparse and contradicting evidence. This article is intended to critically review the available evidence and facilitate patient-specific treatment recommendations based on current studies, guidelines and expert opinion.

\section{Korrespondenzadresse}

Prof. Dr. med. Rupert Bauersachs

Grafenstraße 9

64283 Darmstadt

Tel. 06151 - 1074401

Fax 06151 - 1074429

E-Mail:

Rupert.Bauersachs@mail.Klinikum-Darmstadt.de
The lower leg vein thrombosis - a disease for specialists

Phlebologie 2018; 47: 319-328

https://doi.org/10.12687/phleb2449-6-2018

Eingegangen: 30. Juni 2018

Angenommen: 02. Juli 2018

English version available at:

www.thieme.de/phlebo

\section{Einleitung}

Bei der distalen Venenthrombose, genauer der ,isolierten" distalen Thrombose, handelt es sich um eine (tiefe) Venenthrombose der paarigen Unterschenkelvenen oder Muskelvenen, oder eine Kombination von beiden. Synonym wird die Bezeichnung „Unterschenkelvenenthrombose“ oder „Ein-Etagen-Thrombose“, im Englischen "isolated deep distal vein thrombosis" (IDDVT) oder ,isolated calf vein thrombosis" (ICVT) verwendet. Bei Ausdehnung bis in die Trifurkation, dem Zusammenfluss der Fibular- und Tibialvenen in die V. poplitea spricht man von einer proximalen tiefen Venenthrombose (pTVT) (1). Im Gegensatz zur proximalen Thrombose ist das Therapieregime für die Muskelvenenthrombose oder isolierte distale Unterschenkelthrombose nicht klar definiert (2, 3). Dieser Artikel soll hierüber anhand der Zusammenstellung aktueller Studien, Leitlinien und Expertenmeinungen die Therapieempfehlung kritisch beleuchten und erleichtern.

\section{Studienlage zur distalen Thrombose und Muskelve- nenthrombose}

Das TULIPA-Register (4) analysierte das Risikoprofil von ambulant erworbenen TVT und das diagnostische und therapeutische Management in Deutschland. An diesem Register beteiligten sich fast 400 Praxen, welche insgesamt 4.956 Patienten mit Thromboseverdacht untersuchten. Die Ergebnisse des Registers zeigten, dass bei etwa bei der Hälfte der diagnostizierten Thrombosen isoliert das distale Venensystem betroffen war. Bei fast $50 \%$ der distalen Thrombosen (dTVT) waren die Muskelvenenthrombosen (MVT) alleine oder zusätzlich betroffen. In anderen Untersuchungen wie z.B. der OPTIMEV-Studie (5) zeigte sich ein etwas anderes Verteilungsmuster mit etwa 50\% MVT, 25\% axialen dTVT und ca. 25\% Kombinationen.

Die diagnostische Sicherheit einer kompletten Kompressions-Ultraschalluntersuchung (CCUS), d.h. einschließlich des Unterschenkels erscheint ausreichend zu sein, um eine Antikoagulationstherapie zu beginnen oder abzulehnen (1). Aus der Arbeitsgruppe von Schellong et al. ist bekannt, dass bei negativem CCUS ohne die Initiierung einer oralen Antikoagulation (OAK) bei Patienten nach drei Monaten lediglich $0,57 \%(0,25-0,89 \%)$ venöse Thromboembolien (VTE) auftreten (6). Unter deutschen Alltagsbedingungen war die Rezidivrate, d.h. die "Versagerquote" bei eindeutig negativem CCUS 0,34\% (0,09$0,88 \%)(6,7)$. Diese geringe Rate konnte aber nur bei guten Untersuchungsbedingungen und objektiv eindeutigen Untersuchungsergebnissen erreicht werden $(6,8)$. War der initiale CCUS nicht eindeutig oder lag eine Unsicherheit beim Untersucher vor, ob es sich um eine Thrombose handelt, fanden sich nach drei Monaten deutlich 
mehr venöse Thromboembolien (VTE), nämlich 2,5\% (0,69-6,28\%).

Aus einer aktuellen Übersichtsarbeit zur dTVT von Robert-Ebadi et al. (9), lassen sich wichtige Schlussfolgerungen über das Management der distalen Thrombose ableiten: Auf Basis von mehr als $10.000 \mathrm{~Pa}$ tienten mit TVT-Verdacht bestätigte sich die Diagnose einer TVT bei 23\% der Patienten. Die tatsächlich erkrankten Patienten wiesen in $49 \%$ eine proximale TVT und in $51 \%$ eine dTVT auf. Wurde keine Antikoagulation bei initial negativem CCUS durchgeführt, betrug die 3-Monatsrate an VTE 0,6\%, was den Ergebnissen der Untersuchung von Schellong et al. entspricht (10). Robert-Ebadi et al. konnte zeigen, dass bei Patienten mit negativem CCUS welche nach $168 \pm 25$ Tagen eine klinische Nachuntersuchung erhielten, nur bei zwei Patienten eine tiefe Venenthrombose dokumentiert wurde $(0,9 \%, 95 \%$ CI: $0,1-3,3 \%)$ (9). Zusätzlich wurde in der Studie von Robert-Ebadi et al. untersucht, ob ein Unterschied in der Sensitivität der Labordiagnostik zwischen der proximalen und distalen Thrombose besteht. Die Studie zeigte, dass die Sensitivität des ELISA-D-Dimer-Test für die proximalen TVT höher war als für die distale TVT ( $98 \%$ vs. 86\%) (9). Dieser Unterschied bestätigte sich ebenfalls mit $94 \%$ vs. $79 \%$ für die Latex-Agglutinations-Tests und mit $84 \%$ vs. $64 \%$ für die Testung mit Vollblutagglutination (9). Anzunehmen wäre, dass dieser Unterschied durch die größere Thrombusmenge und damit auch vermehrten Fibrinspaltprodukten bei Lokalisation im Oberschenkel zustande kommt.

Bedacht werden muss aber, dass die Spezifität der D-Dimerbestimmung zu gering ist, um aus einer Erhöhung der Konzentration im Blut allein eine Thrombose zu beweisen. Die einzig sinnvolle Verwendung des D-Dimer-Tests ist die Anwendung nach vorheriger Schätzung der klinischen Wahrscheinlichkeit (KW) z.B. anhand der im Wells-Score definierten Kriterien. Ein negativer D-Dimer Wert bedeutet bei niedriger $\mathrm{KW}$, dass keine therapiebedürftige Thrombose vorliegt und der $\mathrm{Pa}$ tient keine weitere diesbezügliche Diagnostik (2) benötigt . Bei hoher KW hingegen sollten D-Dimer-Tests generell nicht eingesetzt werden, da deren negativ prädiktiver
Wert in dieser Situation nicht ausreichend hoch ist und man im Falle eines negativen Testbefunds möglicherweise eine weitergehende Diagnostik fälschlicherweise unterlassen würde (2, 11-13).

\section{Wie häufig verursacht eine dTVT eine Lungenembolie?}

$\mathrm{Zu}$ dieser Fragestellung wurden in einem aktuellen systematischen Review (14) acht randomisierte Studien und 13 prospektive Kohortenstudien ausgewertet. Es wurde gezeigt, dass die Inzidenz einer Lungenembolie auf Basis einer isolierten distalen Unterschenkelvenenthrombose zwischen $0 \%$ und $6,2 \%$ betrug. Tödliche LE wurden in dieser Untersuchung nicht beobachtet (14). Wenn bei Erstvorstellung bestehende LE mitberücksichtigt wurden, ergaben sich Raten von $5,1 \%$ - 43,4\% (Median 12,8\%); dagegen zeigte sich in 16 Studien, die explizit eine initiale, gleichzeitige LE ausgeschlossen hatten eine LE-Rate von $0-6,2 \%$ (Median 1,1\%). Da weder LE bei asymptomatischen Patienten noch tödliche LE miterfasst wurden, sind die LE-Raten hier unterschätzt. Insgesamt zeigten diese Untersuchungen, dass eine LE nach dTVT selten war und der klinische Schweregrad oft unklar blieb.

Die Daten waren jedoch bezüglich der Patienten, den Diagnosemethoden und dem Follow-up sehr heterogen. So wurden LE mittels Ventilations/Perfusions-Szintigraphie diagnostiziert, wohingegen aktuelle Studien vorwiegend CT-Angiographien verwenden. Beachtet werden sollte auch bei der Interpretation solcher Studienergebnisse die Tatsache, dass bei Nachweis einer LE und isolierter dTVT, die LE aus einer vorbestehenden proximalen TVT entstanden sein könnte und im nachfolgenden Duplex nur noch eine dTVT vorhanden war. Vica versa ergibt sich daraus, dass in den Studien nicht jeder Patient mit dTVT auf eine zusätzliche LE hin mittels CT untersucht wurde. Hervorzuheben ist unter diesem Aspekt auch die Tatsache, dass Patienten mit einer LE in $80 \%$ ein Rezidiv im Lungenstromgebiet erleiden und lediglich bei $20 \%$ eine andere Strombahn als die isolierte periphere Thrombose als Rezidiv betroffen wird $(15,16)$. Die Studie von Dou- ketis et al. bestätigt auch den Umkehrschluss, so dass eine periphere Thrombose in ebenfalls 80\% zu einer Rezidivthrombose ohne LE führt (17).

\section{Aktuelle Leitlinien: Emp- fehlung zur Behandlung von distalen Thrombosen}

In den aktuellen amerikanischen Leitlinien des "American College of Chest Physicians" ACCP (3) wird - mit sehr schwachem Empfehlungsgrad (2C) - für die dTVT ohne schwere Symptome, oder bei hohem Blutungsrisiko und ohne Vorliegen von Risikofaktoren (RF) für eine Thrombusaszension statt einer Antikoagulation ein wiederholter Ultraschall im zeitlichen Abstand von zwei Wochen vorgeschlagen (Grad 2C, also schwache Evidenz). Nur bei schweren Symptomen oder bestehenden RF wird eine Antikoagulation empfohlen (1B) $(\triangleright$ Tab. 1).

Als Risikofaktoren für eine Thrombusausdehnung werden folgende Parameter konstatiert: Positiver D-Dimer Wert, ausgedehnte Thrombose ( $>5 \mathrm{~cm}$ lang, $>7 \mathrm{~mm}$ Durchmesser) oder das Heranreichen des Thrombus an eine proximale Vene. Liegen persistierende auslösende RF, wie ein aktiver Tumor, frühere VTE oder eine Hospitalisierung vor, wird eine Antikoagulation für mindestens drei Monate vorgeschlagen (1B). Im Gegensatz zur Thrombose der axialen tiefen Venen wird für die Muskelvenenthrombose (MVT) ein niedrigeres Risiko für eine Progression angenommen und daher nur eine Kontrolle mittels Duplexuntersuchung nach zwei Wochen vorgeschlagen (2C). Falls sich bei Ultraschallkontrolle eine Thrombusausdehnung in den Unterschenkelvenen zeigt, wird ebenfalls eine Antikoagulation vorgeschlagen $(2 \mathrm{C})$, bei proximaler Ausdehnung wird die Antikoagulation empfohlen (1B). Führt die Konstellation zur Einleitung einer Antikoagulation, entspricht die Dosis und die Dauer der Therapie der akuten proximalen TVT (1C).

Folgt man den interdisziplinären deutschsprachigen S2k Leitlinien der Deutschen Gesellschaft für Angiologie (DGA), sollte ein Patient mit dTVT nicht länger als drei Monate antikoaguliert werden. Diese 
Tab. 1 Aktuelle Leitlinienempfehlungen zur Behandlung der distalen Thrombose; *Hohes Risiko (modifiziert nach [18]): Persistierende RF, unprovoziert, persistierend eingeschränkte Mobilität, Hospitalisierung, männliches Geschlecht, Z.n. VTE, >50 Jahre, Ausdehnung in Trifurkation, >1 Unterschenkelvene betroffen, dTVT in beiden Beinen; * Niedriges Risiko: Transiente RF, Post-OP, Gips, Trauma, Langstreckenreise, Immobilisation, Pille oder Hormonersatztherapie (falls beendet)

\begin{tabular}{|c|c|c|c|}
\hline Gesellschaft & $\begin{array}{l}\text { Deutsche Gesellschaft für Angiologie (DGA) } \\
\text { S2k-LL (2) }\end{array}$ & $\begin{array}{l}\text { European Society for Cardio- } \\
\text { logy (ESC) (18) }\end{array}$ & $\begin{array}{l}\text { American College of Chest Physician } \\
\text { (ACCP) (3) }\end{array}$ \\
\hline $\begin{array}{l}\text { Dauer der Anti- } \\
\text { koagulation }\end{array}$ & $\leq 3$ Monate, auch bei Rezidiv oder idiopathisch & $\begin{array}{l}3 \text { Monate bei hohem* Rezidivri- } \\
\text { siko }\end{array}$ & $\begin{array}{l}\text { MVT mit niedrigerem Risiko eingestuft (2C) } \\
\text { Nur bei schweren Symptomen oder Risikofak- } \\
\text { toren: therapeutische Antikoagulation (1B) }\end{array}$ \\
\hline Anpassung & $\begin{array}{l}\text { Individuell auch kürzer, auch niedrigere Dosis mög- } \\
\text { lich }\end{array}$ & $\begin{array}{l}\text { Bei niedrigem Risiko**: } 4-6 \\
\text { Wochen Therapie }\end{array}$ & $\begin{array}{l}\text { Bei niedrigem Risiko oder keinen Symptomen } \\
\text { keine Antikoagulation sondern Ultraschall } \\
\text { nach } 2 \text { Wochen }(2 C)\end{array}$ \\
\hline Besonderheit & $\begin{array}{l}\text { Wenn Risikofaktoren wie Tumor oder Antiphospho- } \\
\text { lipidsyndrom vorhanden, dann längere AK-Dauer } \\
\text { Keine Unterscheidung zwischen MVT / dTVT }\end{array}$ & $\begin{array}{l}\text { Bei niedrigem Risiko niedrigere } \\
\text { Dosis möglich } \\
\text { oder Überwachung (Ultraschall) }\end{array}$ & $\begin{array}{l}\text { Bei Risikofaktoren } \geq 3 \text { Monate (1B) } \\
\text { - pos. D-Dimere } \\
>5 \mathrm{~cm} \text { Länge } \\
\text { - } 7 \mathrm{~mm} \text { Durchmesser } \\
\text { - Heranreichen an prox. Venen } \\
\text { - Tumor } \\
\text { - Z.n. VTE } \\
\text { - Hospitalisierung } \\
\text { - Hohes Blutungsrisiko (2B) }\end{array}$ \\
\hline
\end{tabular}

Empfehlung wird auch für ein Rezidiv oder eine idiopathische distale Thrombose ausgesprochen (2). Unterschiede zwischen MVT und dTVT werden hierbei nicht gemacht, und im Sinn einer Einzelfallentscheidung bleibt Raum für eine kürzere Behandlungsdauer und/oder reduzierte Dosis des Antikoagulans.

Die 2017 aktualisierten europäischen Leitlinien der European Society of Cardiology (ESC) (18) empfehlen bei hohem Rezidivrisiko eine dreimonatige Antikoagulation und bei niedrigem Risiko die Antikoagulation für 4-6 Wochen entweder in therapeutischer oder reduzierter Dosis ( Tab. 1). Alternativ zur medikamentösen Therapie wird auch die (Ultraschall-) Überwachung ohne Antikoagulation angeboten.

\section{Risikofaktoren für das Auf- treten von dTVT}

In die prospektive, multizentrische Beobachtungsstudie OPTIMEV (Optimizing history taking for evaluating the risk of venous thromboembolism) $(5,19)$ wurden Patienten mit objektiv bestätigter symptomatischer distaler TVT (dDVT) eingeschlossen und mit Patienten welche eine proximale TVT aufwiesen verglichen. Es

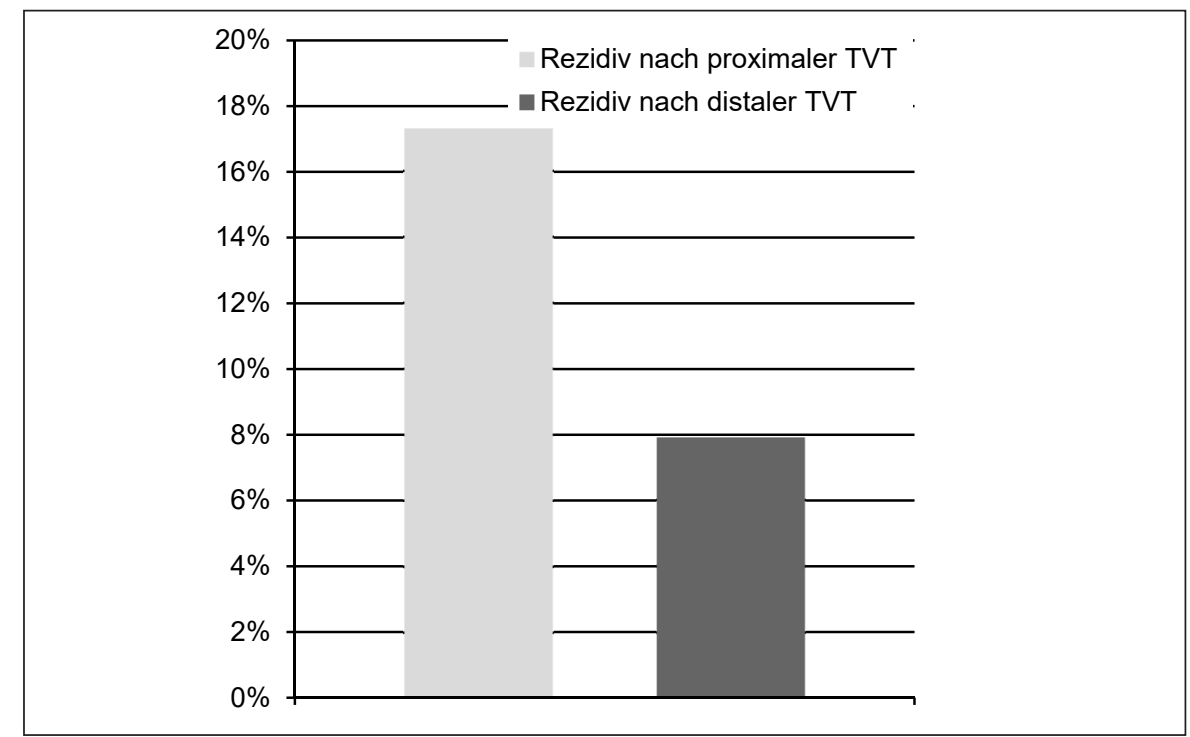

Abb. 1 Der mediane Follow-up (der Überlebenden) betrug 94 Monate nach proximaler TVT, bzw. 84 Monate nach dTVT. Insgesamt wurden bei über 3.175 Patientenjahren 15,0\% rezidivierende symptomatische proximale TVT oder LE beobachtet, davon 17,3\% nach proximaler TVT (rechts) und 7,9\% nach dTVT (links) entsprechend Ereignisraten von 4,5\%/Jahr (3.7-5.4) für die proximalen TVT und 2,0\%/Jahr $(1.1-3.3 \%)$ für dTVT. Insgesamt war die dTVT mit einem niedrigeren Rezidivrisiko vergesellschaftet [HR $0,49(0.32-0.74)]$.

wurde gezeigt, dass bei den 6.141 Patienten mit Thromboseverdacht eine isolierte distale Thrombose signifikant häufiger $\mathrm{zu}$ finden war als eine proximale DVT (56,8\% vs. $43,2 \%$; $=0,01)$. Die dTVT war häufiger assoziiert mit passageren Risikofaktoren, wie einer kürzlich durchgeführten OP oder einer Gipsimmobilisation; auch eine kürzlich durchgeführte Reise wurde als Risikofaktor gehäuft gezählt $(5,19)$.

Die proximale TVT war hingegen signifikant häufiger mit chronischen Erkrankungen wie einem Tumor, einer kardialen oder respiratorischen Insuffizienz sowie 
Tab. 2 Risikofaktoren und deren Bewertung für das Auftreten einer proximalen oder einer distalen Thrombose, sowie deren Korrelation zueinander dar. Als Kontrollpatienten wurden Patienten gewählt, bei denen das entsprechende Kriterium nicht vorlag. Ergebnisse aus der OPTIMEV-Studie (19).

\begin{tabular}{|c|c|c|c|}
\hline Risikofaktoren & $\begin{array}{l}\text { Proximale TVT } \\
\text { vs. Kontrollpa- } \\
\text { tienten } \\
\text { OR [CI 95\%] }\end{array}$ & $\begin{array}{l}\text { Distale TVT } \\
\text { vs. Kontrolle } \\
\text { OR [CI 95\%] }\end{array}$ & $\begin{array}{l}\text { Distale DVT } \\
\text { vs. proximale TVT } \\
\text { OR [Cl 95\%] }\end{array}$ \\
\hline Kürzlicher Gips (Ja vs. Nein) & $2.6[1.5-4.4]$ & $5.4[3.9-7.7]$ & $2.2[1.3-3.8]$ \\
\hline Kürzliche OP (Ja vs. Nein) & $1.3[1.0-1.8]$ & $2.3[1.9-2.9]$ & $1.8[1.3-2.5]$ \\
\hline $\begin{array}{l}\text { Kürzliche Langstreckenreise } \\
\text { (Ja vs. Nein) }\end{array}$ & $2.1[1.2-3.6]$ & $4.1[2.8-6.2]$ & $1.7[1.0-2.8]$ \\
\hline Varikosis (Ja vs. Nein) & $0.7[0.5-0.9]$ & $0.9[0.8-1.1]$ & $1.3[1.0-1.7]$ \\
\hline $\begin{array}{l}\text { Herz- / respiratorische Insuffi- } \\
\text { zienz (Ja vs. Nein) }\end{array}$ & $3.0[2.1-4.4]$ & $1.5[1.0-2.2]$ & $0.6[0.4-0.9]$ \\
\hline Alter $>75$ vs. $<50$ & $2.0[1.5-2.6]$ & $1.1[0.9-1.4]$ & $0.5[0.4-0.7]$ \\
\hline $\begin{array}{l}\text { Aktive Krebserkrankung } \\
\text { (Ja vs. Nein) }\end{array}$ & $3.2[2.5-4.1]$ & $1.5[1.2-1.9]$ & $0.5[0.4-0.7]$ \\
\hline
\end{tabular}

mit einem Alter > 75 Jahre assoziiert ( $\triangleright$ Tab. 2). Die Mehrzahl der Patienten (96,8\%) mit dTVT erhielt eine Antikoagulationstherapie. Trotz der Tatsache, dass es keinen Unterschied im Auftreten von Rezidiven und schweren Blutungen gab, war die Mortalitätsrate bei Patienten mit proximaler TVT signifikant höher als bei Patienten mit isolierter distaler DVT ( 8,0 vs. $4,4 \%$; $<0,01)(19)$.

\section{Risikofaktoren für das Auf- treten von Rezidiv-VTE nach dTVT}

In der bereits erwähnten OPTIMEV-Studie (5) wurde drei Jahre nach Index-VTE und nach Beendigung der Antikoagulation die Inzidenz von VTE-Rezidiven bei Patienten ohne Tumorerkrankung nach einer ersten dTVT mit der Inzidenz initial proximaler TVT verglichen. Diese Analyse ergab, dass bei dTVT im Vergleich zu proximaler TVT eine niedrigere annualisierte VTE-Rezidivrate bestand. Interessant war dabei aber, dass beide Gruppen vergleichbare Raten an Lungenembolien aufwiesen: $1,0 \%$ $(0,5-2,3 \%)$ vs. $0,9 \%(0,5-1,6 \%) ; \mathrm{p}=0,83)$. Zudem hatten Patienten mit primärer dTVT als Rezidiv häufiger ebenfalls eine dTVT und im Umkehrschluss die Patienten mit initialer proximaler TVT als Rezidiv wieder eine proximale TVT ( $\triangleright$ Tab. 3 ) (5).
Aus der Tabelle 3 ist zu entnehmen, dass a) Alter über 50 Jahre, b) eine unprovozierte dTVT, c) die Thrombosierung von mehr als einer Vene in einem Bein oder d) Thrombosen in beiden Beinen das Rezidivrisiko verdreifachten. Bei allen Faktoren liegt das Rezidivrisiko jeweils über 3\% pro Patientenjahr. Interessanterweise beeinflusste weder das Geschlecht noch das Vorliegen einer Muskelvenenthrombose, oder der Befall von tiefen Unterschenkelvenen, sowie der Durchmesser des Thrombus oder eine Dauer der Antikoagulation über 90 Tage das Rezidivrisiko.

\section{Rezidiv-VTE und Überleben bei Patienten mit distaler TVT im Vergleich zu Patien- ten mit proximaler TVT}

In einer retrospektiven Analyse wurden von 2000-2012 konsekutive Patienten mit akuter distaler und proximaler TVT ohne begleitende LE ausgewertet (20). Diese Studie zeigte, dass dTVT ein geringeres Rezidivrisiko haben als pTVT. Für diese Analyse wurden aus insgesamt 4.759 Datensätzen 831 Patienten eingeschlossen. Von diesen hatten 629 Patienten eine symptomatische pTVT, 202 eine dTVT, davon wiederum $49,6 \%$ eine MVT und 27,1\% eine Thrombose der tiefen Unterschenkelvenen. Bei 13,4\% wurde sowohl eine MVT als
Tab. 3 Risikofaktoren nach Beendigung der Antikoagulation für Rezidivthrombosen bei initialer distaler Thrombose im Vergleich zu Patienten, bei denen das Kriterium nicht vorliegt. Daten aus der prospektiven, multizentrischen Beobachtungsstudie OPTIMEV (5).

\begin{tabular}{l|l|l|}
\hline Risikofaktor & $\begin{array}{l}\text { Multivariate } \\
\text { HR }\end{array}$ & $\begin{array}{l}\text { Rezidiv-VTE } \\
\text { (\%/Patienten- } \\
\text { jahre) }\end{array}$ \\
\hline Bilaterale TVT & $4.0(1.4-11.1)$ & $8.9(3.7-21.4)$ \\
\hline $\begin{array}{l}\text { Multiple uni- } \\
\text { lateral TVT }\end{array}$ & $2.9(1.4-6.1)$ & $4.9(3.1-7.8)$ \\
\hline $\begin{array}{l}\text { Unprovozier- } \\
\text { te TVT }\end{array}$ & $3.1(1.4-6.9)$ & $3.8(2.6-5.6)$ \\
\hline $\begin{array}{l}\text { Alter }>50 \\
\text { Jahre }\end{array}$ & $3.7(1.0-10.6)$ & $3.8(2.6-5.5)$ \\
\hline
\end{tabular}

auch eine Thrombose der tiefen Venen, oder eine dTVT beider Beine diagnostiziert. Die Patienten erhielten eine Antikoagulationstherapie in Dauer und Dosis entsprechend den jeweils gültigen Leitlinienempfehlungen (20).

Insgesamt wurden nach einem Followup von acht Jahren bei 15,0\% rezidivierende symptomatische proximale TVT oder LE beobachtet, davon $17,3 \%$ nach proximaler TVT und 7,9\% nach dTVT entsprechend Ereignisraten von 4,5\%/Jahr für die proximalen TVT und 2,0\%/Jahr für dTVT. Insgesamt war die dTVT mit einem niedrigeren Rezidivrisiko vergesellschaftet. Die Gesamtmortalität lag bei $31,6 \%$, entsprechend 4,8\%/Jahr. Von den 263 verstorbenen Patienten hatten 52 Patienten eine dTVT, entsprechend 25,7\% aller dTVT-Patienten und 211 eine proximale TVT (33,5\% der Gesamtzahl der proximalen TVT) ( Abb. 2). Diese Kohortenstudie zeigt, dass Patienten mit einer ersten dTVT ein signifikant niedrigeres Rezidiv-VTE Risiko aufweisen als solche mit pTVT. Frauen waren in der Gruppe mit dTVT überrepräsentiert ( $56,4 \%$ vs. $48,6 \%$ bei proximalen TVT), ebenso einige auslösende Faktoren (wie z.B. OP oder ein Trauma) (20). 


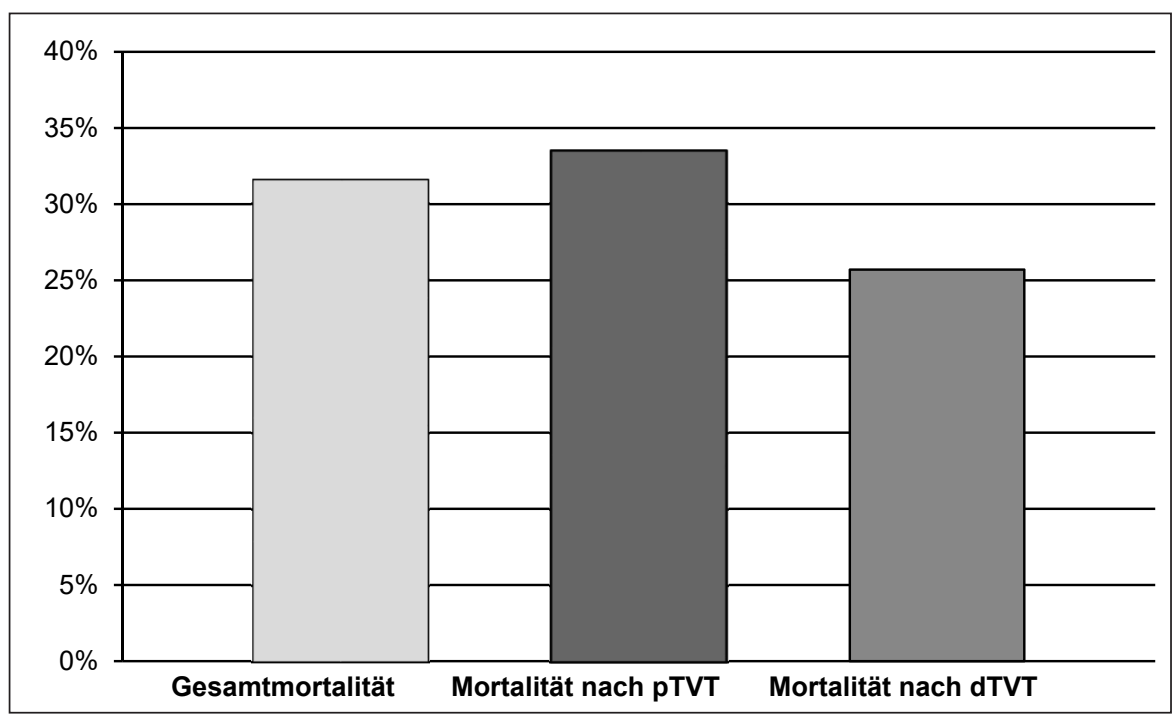

Abb. 2 Die Gesamtmortalität lag bei 31,6\%, entsprechend 4,8\%/Jahr. Von den 263 verstorbenen Patienten hatten 52 eine dTVT, entsprechend $25,7 \%$ aller dTVT-Patienten und 211 eine proximale TVT (33,5\% der Gesamtzahl der proximalen TVT), entsprechend einer HR von 0,75 $(0,55-1,02)$. *Der mediane Follow-up (der Überlebenden) betrug 94 Monate nach proximaler TVT, bzW. 84 Monate nach dTVT. Daten aus einer Kohortenstudie (20).

\section{Tumor-assoziierte distale TVT}

Obwohl die isolierte dTVT häufig mit einem Tumorleiden assoziiert ist, wurde der Langzeitverlauf nach tumorassoziierter dTVT bislang nicht untersucht. In der prospektiven multizentrischen Beobachtungsstudie OPTIMEV (21) wurde nach drei Jahren die Inzidenz von Tod, VTE-Rezidiv und schweren Blutungen bei tumorassoziierter dTVT mit tumorassoziierter proximaler TVT verglichen. Die beiden Gruppen waren durch Propensity Score Matching bezüglich Alter und Geschlecht gleichverteilt. So zeigte die Studie, dass die 92 Patienten mit dTVT ein vergleichbares Risiko für Tod und schwere Blutungen aufwiesen wie die 92 Patienten mit proximaler TVT, allerdings ein höheres VTE-Rezidivrisiko hatten ( $\triangleright$ Abb. 4).

Im Vergleich zu dTVT-Patienten ohne Tumor hatten die dTVT-Tumorpatienten ein 9-fach höheres Risiko für Tod

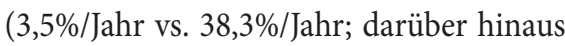
auch ein höheres Risiko für schwere Blutungen $(1,8 \% / \mathrm{Jahr}$ vs. $3,6 \% / \mathrm{Jahr}$ und für VTE-Rezidive (21).

\section{Placebo-Studie bei distaler TVT mit niedrigem Risiko CACTUS}

Zur Therapie der dTVT gibt es insgesamt fünf randomisierte Studien, einige davon sind zum Teil über 30 Jahre alt, mit Vitamin-K-Antagonisten (VKA) durchgeführt und damit der heutigen Wirklichkeit nicht mehr entsprechend (22-24).

Lediglich die CACTUS-Studie (25) bringt wichtige neue Aussagen zum Management der dTVT. Es handelt sich dabei um eine randomisierte placebokontrollierte Studie, die in 23 Zentren in Kanada, Frankreich und der Schweiz ambulante Patienten mit einer ersten dTVT und einem niedrigem Rezidivrisiko, also Patienten ohne ein aktive Tumorerkrankung oder eine vorausgegangene VTE) eingeschlossen hatte. Die 259 eingeschlossenen Patienten erhielten über den Zeitraum von sechs Wochen entweder 171 IE/kg Nadroparin (122 Patienten) oder Placebo (130 Patienten). Zudem erhielten alle Patienten Kompressionsstrümpfe und wurden 90 Tage nachbeobachtet. Leider verzögerte sich die Patientenrekrutierung in der CACTUS-Studie erheblich, so dass von Februar 2008 bis November 2014 nur 259 Patienten (was der
Hälfte der geplanten Patienten entspricht) für die Studie rekrutiert wurden. Aufgrund des placebokontrollierten Designs mit schleppender Rekrutierung wurde die Studie im November 2014 vorzeitig abgebrochen.

Der primäre Endpunkt umfasste Ausdehnung in die proximalen Venen, eine kontralaterale proximale TVT oder eine LE nach sechs Wochen. Es gab keinen signifikanten Unterschied zwischen den beiden Gruppen im primären Endpunkt. Dieser Endpunkt wurde in 3,3\% unter Nadroparin und in 5,4\% in der Placebogruppe erreicht $(\mathrm{p}=0,54)(25)$. Blutungen traten bei fünf Patienten (4\%) in der Nadroparin-Gruppe und bei keinem Patienten in der PlaceboGruppe auf (was einem Risikounterschied von 4,1 entspricht: 95\% Konfidenzintervall $0,4-9,2 ; p=0,0255)$. Im Nadroparinarm starb ein Patient an einem metastasierten Tumorleiden und bei einem Patienten wurde eine HIT II diagnostiziert (25). Mit diesem Studienergebnis konnte bei einem ambulanten Niedrigrisiko-Kollektiv mit dTVT durch die Applikation von Nadroparin im Vergleich zu Placebo weder eine proximale Thromboseausdehnung noch eine Verhinderung von VTE- Ereignissen erreicht werden. Es kam jedoch unter Nadroparin erwartungsgemäß zu einer $\mathrm{Zu}$ nahme des Blutungsrisikos (25).

\section{Wie soll eine distale Thrombose antikoaguliert werden?}

Zur Beantwortung der Fragen, welche therapeutische Relevanz ist der Antikoagulation einer distalen Venenthrombose zuzuordnen und die Dauer der Antikoagulation herauszuarbeiten hat Franco et al. 2017 eine interessante Metaanalyse durchgeführt (26): In dieser Auswertung von randomisierten und Kohorten-Studien war der primärer Endpunkt eine Rezidiv-VTE, einschließlich proximaler TVT-Ausdehnung und LE.

\section{Antikoagulation versus keine Anti- koagulation}

Hierzu wurden 20 Studien mit insgesamt 2.936 Patienten ausgewertet. Fünf Studien 
waren randomisierte kontrollierte Studien und jeweils sieben waren prospektive und sieben retrospektive Studien. Unterschiede bestanden dabei jedoch z.B. im Patientenkollektiv: Fünf Studien fokussierten auf chirurgische Patienten, fünf auf MVT, jeweils drei Studien auf stationäre oder ambulante Patienten. Die meisten Studien verwendeten Ultraschall zur Thrombosediagnostik, einige Studien auch die Phlebographie oder andere Methoden. In fünf Studien bestand die antithrombotische Behandlung aus einer prophylaktischen und in 14 der Studien aus einer therapeutischen Dosis. Auch die Therapiedauer variierte zwischen vier Wochen und zwei Jahren. Allein aus dieser Tatsache erklärt sich die Schwierigkeit die Studienergebnisse auf den klinischen Alltag zu übertragen.

Dennoch konnte die gepoolte Analyse der 20 Studien zeigen, dass durch die Antikoagulation eine signifikante Reduktion von Rezidiv-VTE erreicht werden konnte (6,5\% vs. $12 \%)$ (26). Betrachtet man nur die 16 Studien mit therapeutischer Antikoagulation zur Frage der Notwendigkeit einer Antikoagulation bei der dTVT, so zeigte sich auch hierbei der Vorteil der Antikoagulation in der Verhinderung der VTERezidive (6,5\% vs. $12,4 \%)$. Allerdings zeigte sich eine erhebliche Heterogenität, die vor allem auf die Kohortenstudien zurückzuführen war. Auch die Rate an Lungenem- bolien war bei antikoagulierten Patienten niedriger als in der Kontrollgruppe (1,4\% vs. 2,4\%) ( Abb. 3) (26).

Einige der Studien hatten Blutungskomplikationen entweder gar nicht oder nicht standardisiert angegeben, so dass nur bei neun der Studien (entsprechend 1.385 Patienten) die Blutungsrate ausgewertet werden konnte. Die Gesamtblutungen waren unter der Antikoagulation zwar doppelt so hoch wie ohne Antikoagulation (6,3\% vs. $2,8 \%$ ), wobei sich aber die Rate der schweren Blutungen zwischen den Gruppen nicht unterschied ( $0,4 \%$ vs. $0,7 \%)$ ( $\triangleright$ Abb. 3).

Eine Schwäche dieser Auswertung liegt jedoch in der niedrigen methodologischen Qualität und Heterogenität der Studien: So werteten nicht alle Studien symptomatische Ereignisse aus und die eingesetzte Antikoagulationstherapie war sehr heterogen. Zudem variiert die Therapie zwischen Vitamin K-Antagonisten (Warfarin und Phenprocoumon), niedermolekularem Heparin in therapeutischer oder prophylaktischer Dosis und subkutanen Xa-Inhibitoren. In keiner Studie waren DOAKs verwendet worden. Eine ebenso große Variabilität bestand in der Verwendung von Kompressionsstrümpfen oder den Angaben über den Einsatz von Aspirin oder nicht steroidalen Antiphlogistika (NSAR).
Wie lang sollte antikoaguliert werden: 6 Wochen oder 6 Monate?

Vier Studien (27-30) mit insgesamt 1.136 Patienten verglichen eine kurze AK (sechs Wochen) mit einer langen Antikoagulationsperiode; drei Studien für drei Monate $(27,29)$ und eine Studie für sechs Monate (30). Die gepoolte Auswertung der Studien (26) zeigte eine Reduktion der Rezidivthrombosen unter verlängerter $\mathrm{AK}$ im Vergleich zu den Studien ohne AK (OR 0,50, 95\% CI 0,31-0,79), ohne das Risiko für schwere Blutungen (OR 0,64, 95\% CI 0,15-2,73) signifikant zu erhöhen. Auch die LE waren unter AK deutlich seltener (OR 0,48, 95\% CI 0,25-0,91). Unter Berücksichtigung der verschiedenen Blutungsdefinitionen ergaben sich für die lange Antikoagulationsperiode zwar eine erhöhte Gesamtblutungsraten - schwere Blutungsereignisse waren allerdings nicht signifikant erhöht.

\section{Diskussion}

Die Datenlage aus welcher sich eine Therapie, die Dosis und die Dauer der Antikoagulation bei Patienten mit distaler Thrombose (dTVT) ableiten lässt ist inhomogen und die Empfehlungen variieren zwischen reiner Beobachtung ohne AK

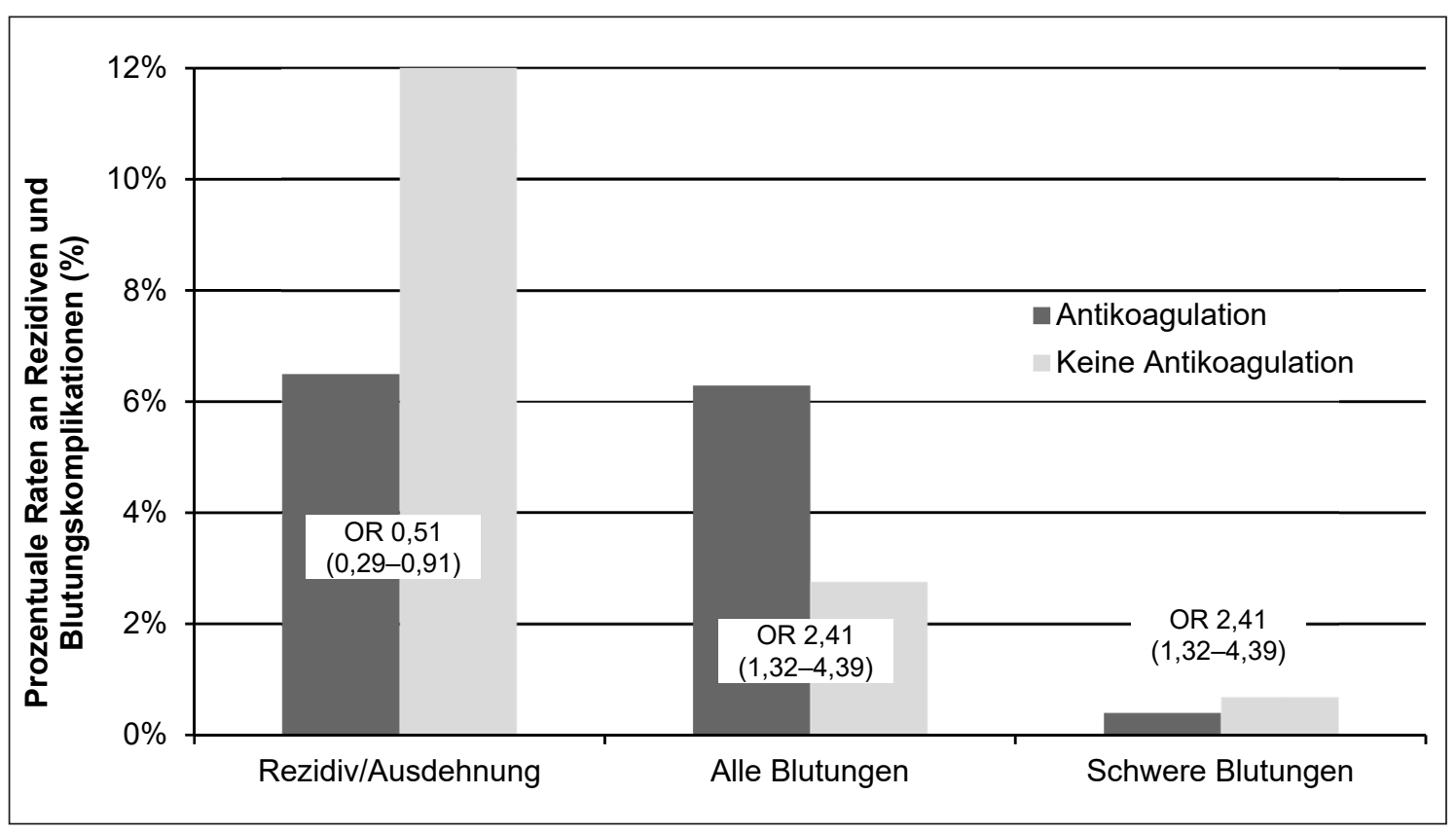

Abb. 3

VTE Rezidive und Thromboseausdehnung, sowie Blutungskomplikationen nach distalen TVT unter prophylaktischer oder therapeutischer Antikoagulation im Vergleich zu keiner Antikoagulation. Daten aus einer Metaanalyse (26). 


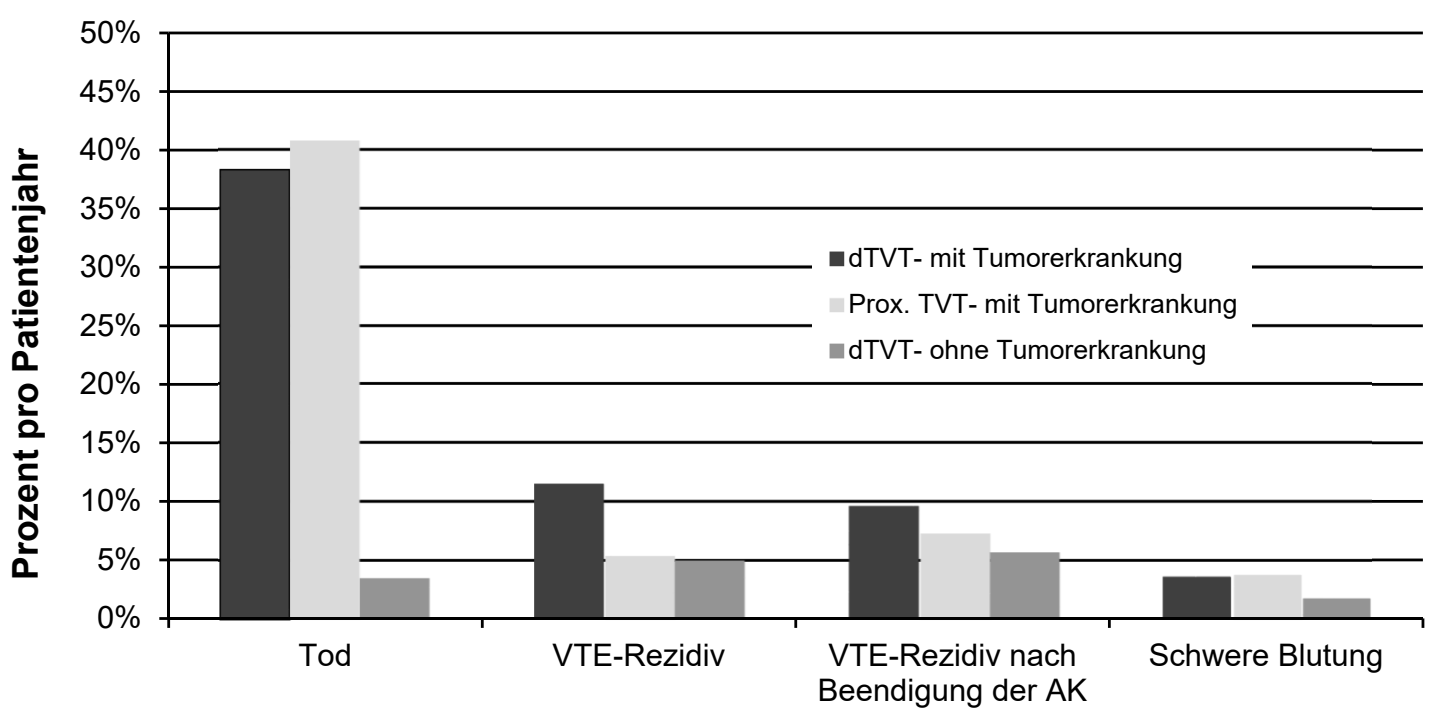

Abb. 4 Graphische Darstellung des Outcome nach distaler und proximaler TVT bei maligner Grunderkrankung: Von links nach rechts werden Mortalität, die VTE-Rezidive, VTE-Rezidive nach Beendigung der AK und das Auftreten von schweren Blutungen bei dTVT-Patienten mit und ohne Tumorerkrankung verglichen und mit dem Outcome der proximalen TVT gegenübergestellt. Daten aus der prospektiven, multizentrischen OPTIMEV-Beobachtungsstudie (21).

und $\mathrm{AK}$ in therapeutischer Dosis teilweise auch länger als drei Monate. Dagegen besteht für proximale Thrombosen ein klares Regime aus therapeutischer Dosis für mindestens 3-6 Monate (2, 18, 27, 31). Die Fülle der kürzlich publizierten Daten zur dTVT ergibt jetzt erstmals eine klare Einordnung dieses häufigen, aber bislang in den großen Studien unterrepräsentierten Krankheitsbildes. Insbesondere erlauben die Daten eine verlässlichere Risikoeinteilung und eine bessere Nutzen-Risiko-Bewertung der Antikoagulation beim Vorliegen einer dTVT. Gerade bei maligner Grunderkrankung haben distale Thrombosen einen noch wichtigeren Stellenwert und müssen daher unbedingt richtig diagnostiziert und behandelt werden $(21,32$, 33).

Aus unserer Sicht ist die hier aufgeführte Metaanalyse von Franco et al. (26) von großer Bedeutung, da in dieser die Datensätze von 20 Studien mit über 4.000 Patienten analysiert wurden. Gezeigt werden konnte, dass bei der distalen TVT eine AK, sowohl in prophylaktischer wie auch in therapeutischer Dosis die Rezidivrate von Thrombosen verringert und das Risiko für eine Lungenembolie um 50\% reduziert. Die Blutungsrate war zwar unter Antikoagulation erhöht, allerdings zeigten sich keine signifikanten Unterschiede bezüglich der schweren Blutungen. So stellten die Autoren dem Risiko einer Lungenembolie von 1,4\% unter fehlender Antikoagulation dem Risiko einer schweren Blutung zu erleiden $(0,4 \%)$ gegenüber. Die Schlussfolgerung der Autoren, dass DOAKs wegen verbessertem Nutzen-Risiko-Verhältnis, Praktikabilität und oraler Anwendbarkeit erwogen werden sollten, ist allerdings durch die Daten für die distale TVT noch nicht belegt.

Die CACTUS-Studie (14) wurde aufgrund fehlender Fördermittel, Auslaufen der Studienmedikation und langsamer Rekrutierung abgebrochen, nachdem weniger als die Hälfte der geplanten Patienten eingeschlossen wurden. Angesichts des Placeboarms wurden Patienten mit höherem Risiko ausgeschlossen; diese Tatsache lässt vermuten, dass Studienärzte möglicherweise Patienten mit mutmaßlich erhöhtem Risiko nicht eingeschlossen hatten. Bei limitierter statistischer Aussagekraft lässt sich nach den Ergebnissen diskutieren, ob Niedrigrisiko-Patienten mit dTVT überhaupt eine Antikoagulation erhalten müssen. Diese Patientenklientel und Patienten mit hohem Blutungsrisiko sollten nicht antikoaguliert werden, sondern nach einer bzw. zwei Wochen oder bei Beschwerdeprogression mittels Ultraschall nachuntersucht werden. Diese Strategie ist jedoch meist ambulant nicht praktikabel. So könnten für diese Patienten in Zukunft niedrige Dosen von NMH, Fondaparinux oder DOAK für etwa 40 Tage angeboten werden, entsprechend dem Regime der oberflächlichen Venenthrombose in der CALISTO (34) oder SURPRISE-Studie (35).

Die OPTIMEV Studie zeigte, dass hingegen Tumorpatienten mit dTVT ein hohes Risiko für VTE-Rezidive aufweisen (21), Tumorpatienten mit dTVT eine ähnliche schlechte Prognose wie solche mit einer proximalen TVT haben, und eine wesentlich schlechteres Outcome als dTVT ohne Tumorerkrankung zeigen. Dies unterstreicht die klinische Bedeutung von tumorassoziierten dTVT.

\section{Fazit für die Praxis}

Während für proximale Thrombosen ein klares Regime aus therapeutischer Dosis für mindestens 3-6 Monaten (2, 18, 27, 31) besteht, variieren die Empfehlungen zur Behandlung der dTVT zwischen reiner Beobachtung ohne AK bis hin zur AK in therapeutischer Dosis sogar über drei Monate hinaus.

Die unterschiedlichen Empfehlungen und Unsicherheiten im Management resultieren aus einer wesentlich größeren Hete- 
rogenität der dTVT im Vergleich zur proximalen TVT in vielerlei Hinsicht:

1. Pathophysiologisch ist der Verlauf bei dTVT heterogener und häufiger abortiv so dass sich transiente oder persistierende RF stärker auf eine Progression auswirken. Dagegen ist bei proximaler Thrombose durch die erfolgte Ausdehnung in die V. poplitea ein klares Überwiegen der prothrombotischen Faktoren dokumentiert.

2. Diagnostisch ist die dTVT durch die erforderliche Untersuchung einer wesentlich größeren Zahl von Venen (Vv. tibiales posteriores, Vv. fibulares und Vv. tib. anteriores, sowie bis zu 20 Muskelvenen) deutlich anspruchsvoller als die proximale Thrombose.

3. Differenzialdiagnostisch bedeutet die schlechtere Spezifität des CCUS bei dTVT [94 - 98\% (19)], dass in $2-6 \%$ falsch positive Befunde erhoben werden und eine unnötige AK erfolgt.

4. Akutkomplikationen der AK sind bei Verwechslung mit anderen Erkrankungen wie z.B. Muskelfaserriss, rupturierter Bakerzyste oder Muskeltrauma erhöht.

5. Das Risiko/Nutzen Verhältnis der $\mathrm{AK}$ und damit die Indikationsstellung zur AK variiert erheblich und ist oftmals schwieriger zu erheben als bei proximaler TVT.

6. Die Mortalitätsrate der dTVT bewegt sich in einem wesentlich breiteren Bereich als bei der proximalen TVT und ist daher schwieriger abzuschätzen.

7. Das Rezidivrisiko der dTVT ist sehr heterogen und daher auch die Notwendigkeit und Dauer einer AK; dies reicht von sehr niedrigem Risiko bei klaren Auslösern bis hin zu sehr hohem Risiko, z.B. bei Tumorerkrankungen.

8. Die Bewertung der Risikofaktoren ist damit bei dTVT wesentlich bedeutsamer und schwieriger als bei proximaler TVT.

Die dTVT ist das komplexere, fehlerträchtigere Krankheitsbild verglichen mit der proximalen TVT, und das Management ist deutlich anspruchsvoller. Das Management der dTVT gehört daher in die Hand des Spezialisten, um falsche Abschätzungen und Fehlentscheidungen $\mathrm{zu}$ vermeiden.
Die dTVT stellt damit hohe Anforderungen an den behandelnden Arzt.

\section{Konsequenzen für das praktische Management}

- Standarddiagnostik für die Diagnostik der distalen TVT ist in Deutschland der Kompressionsultraschall (CCUS).

- Bei niedrigem Rezidivrisiko ( Tab. 1) kann der CCUS ohne Antikoagulation nach zwei Wochen oder bei Beschwerdeprogredienz wiederholt werden, und dies als Management ausreichen; zusätzlich könnte eine Thromboseprophylaxe eingesetzt werden, wenn ein transienter Risikofaktor (z.B. eingeschränkter Mobilität) dokumentiert wird.

- Alternativ kann eine Antikoagulation über einen begrenzten Zeitraum (auch kürzer als drei Monate) erfolgen; die Leitlinien lassen auch niedrigere als voll-therapeutische Dosierungen $\mathrm{zu}$.

- Es muss eine sorgfältige Abklärung von Differentialdiagnosen, wie z.B. Muskelfaserriss, Bakerzyste oder Einblutung vor Beginn einer Antikoagulation erfolgen.

- Bei hohem Blutungsrisiko oder Unsicherheit in der Befunderhebung sollte eine Folgeuntersuchung (CCUS) nach einer Woche erfolgen. Auch hier könnte der Einsatz einer vorübergehenden Thromboseprophylaxe nach individueller Abwägung bei zusätzlichem transienten Risikofaktor sinnvoll sein.

- Bei hohem Thrombose-Rezidivrisiko ( $>$ Tab. 1) wird eine volle Therapiedosis über drei Monate, kongruent der Behandlung einer proximalen TVT empfohlen. Ein Patient mit dTVT sollte nicht länger als drei Monate antikoaguliert werden. Diese Empfehlung wird auch für das Rezidiv oder unprovozierte distale Thrombose ausgesprochen (2, 31, 36).

- Patienten mit einem Antiphospholipidsyndrom, aktivem Tumor oder fortbestehenden Risikofaktoren sollten länger als drei Monate antikoaguliert werden.

- Ob bei der dTVT der Einsatz von niedrig dosierten DOAKs der herkömmlichen Antikoagulation mit NMH oder Fondaparinux (Xa-Inhibitor) gleichwertig oder überlegen ist, wurde bislang nicht untersucht.
Interessenkonflikt

Nach Angaben der Autoren bestehen keine Interessenkonflikte.

\section{Ethische Richtlinien}

Für das Manuskript wurden keine Studien an Menschen oder Tieren durchgeführt.

\section{Literatur}

1. Schellong SM. [Ultrasound investigation of vessels supplying the extremities]. Der Radiologe. 2009 Nov;49(11):1005-15. PubMed PMID: 19859687. Ultraschalluntersuchung der extremitatenversorgenden Gefäße.

2. Hach-Wunderle V, Gerlach H, Konstantinides S, Noppeney T, Riess H, Schellong S, et al. Interdisziplinäre S2k: Leitlinie: Diagnostik und Therapie der Bein- und Beckenvenenthrombose und der Lungenembolie; Registernummer 065 - 002. VASA 2016; 45(Suppl. 90): 1-48.

3. Kearon C, Akl EA, Ornelas J, Blaivas A, Jimenez D, Bounameaux H, et al. Antithrombotic Therapy for VTE Disease: CHEST Guideline and Expert Panel Report. Chest. 2016 Feb;149(2):315-52. PubMed PMID: 26867832. Epub 2016/02/13. eng.

4. Schellong S, Gerlach H, Hach-Wunderle V, Rabe E, Riess H, Carnarius H, et al. Diagnostic WorkUp And Diagnostic Safety In Patients With Suspected Deep Vein Thrombosis - Data From The German Tulipa Registry. Journal of Thrombosis and Hemostasis 2007; 5(Suppl. 2).

5. Galanaud JP, Sevestre MA, Genty C, Kahn SR, Pernod G, Rolland C, et al. Incidence and predictors of venous thromboembolism recurrence after a first isolated distal deep vein thrombosis. Journal of thrombosis and haemostasis : JTH. 2014 Apr; 12(4): 436-443. PubMed PMID: 24450376.

6. Schellong SM, Schwarz T, Halbritter K, Beyer J, Siegert G, Oettler W, et al. Complete compression ultrasonography of the leg veins as a single test for the diagnosis of deep vein thrombosis. Thromb Haemost 2003 Feb; 89(2): 228-234. PubMed PMID: 12574800. Epub 2003/02/08. eng.

7. Schellong SM, Gerlach H, Hach-Wunderle V, Rabe E, Riess H, Carnarius H, et al. Diagnosis of deepvein thrombosis: adherence to guidelines and outcomes in real-world health care. Thromb Haemost. 2009 Dec;102(6):1234-40. PubMed PMID: 19967156. Epub 2009/12/08. eng.

8. Gibson NS, Schellong SM, Kheir DY, Beyer-Westendorf J, Gallus AS, McRae S, et al. Safety and sensitivity of two ultrasound strategies in patients with clinically suspected deep venous thrombosis: a prospective management study. Journal of thrombosis and haemostasis. JTH 2009 Dec; 7(12): 2035-2041. PubMed PMID: 19817986. Epub 2009/10/13. eng.

9. Robert-Ebadi H, Righini M. Management of distal deep vein thrombosis. Thrombosis research. 2017 Jan;149:48-55. PubMed PMID: 27889688. Epub 2016/11/28. eng. 
10. Schellong SM, Schwarz T, Pudollek T, Schmidt B, Schroeder HE. Complete compression ultrasound for the diagnosis of proximal and distal deep venous thrombosis--a retrospective outcome study. VASA Zeitschrift fur Gefasskrankheiten 2001 Nov; 30(4): 253-257. PubMed PMID: 11771208.

11. Geersing GJ, Janssen KJ, Oudega R, Bax L, Hoes AW, Reitsma JB, et al. Excluding venous thromboembolism using point of care D-dimer tests in outpatients: a diagnostic meta-analysis. Bmj. 2009 Aug 14;339:b2990. PubMed PMID: 19684102. Pubmed Central PMCID: 2727580.

12. Prisco D, Grifoni E. The role of D-dimer testing in patients with suspected venous thromboembolism. Seminars in thrombosis and hemostasis. 2009 Feb;35(1):50-9. PubMed PMID: 19308893.

13. Stein PD, Hull RD, Patel KC, Olson RE, Ghali WA, Brant $\mathrm{R}$, et al. D-dimer for the exclusion of acute venous thrombosis and pulmonary embolism: a systematic review. Annals of internal medicine 2004 Apr 20; 140(8): 589-602. PubMed PMID: 15096330.

14. Wu AR, Garry J, Labropoulos N. Incidence of pulmonary embolism in patients with isolated calf deep vein thrombosis. Journal of vascular surgery Venous and lymphatic disorders 2017 Mar; 5(2): 274-279. PubMed PMID: 28214497. Epub 2017/02/20. eng.

15. Kearon C. Extended anticoagulation for unprovoked venous thromboembolism: a majority of patients should be treated. J Thromb Thrombolysis. 2011 Apr;31(3):295-300. PubMed PMID: 21331558. Epub 2011/02/19. eng.

16. Schulman S, Lindmarker P, Holmstrom M, Larfars G, Carlsson A, Nicol P, et al. Post-thrombotic syndrome, recurrence, and death 10 years after the first episode of venous thromboembolism treated with warfarin for 6 weeks or 6 months. Journal of thrombosis and haemostasis JTH. 2006 Apr;4(4):734-42. PubMed PMID: 16634738.

17. Douketis JD, Kearon C, Bates S, Duku EK, Ginsberg JS. Risk of fatal pulmonary embolism in patients with treated venous thromboembolism. Jama. 1998 Feb 11; 279(6): 458-462. PubMed PMID: 9466640.

18. Mazzolai L, Aboyans V, Ageno W, Agnelli G, Alatri A, Bauersachs R, et al. Diagnosis and management of acute deep vein thrombosis: a joint consensus document from the European society of cardiology working groups of aorta and peripheral circulation and pulmonary circulation and right ventricular function. European heart journal $2017 \mathrm{Feb}$ 17. PubMed PMID: 28329262.

19. Galanaud JP, Sevestre-Pietri MA, Bosson JL, Laroche JP, Righini $\mathrm{M}$, Brisot $\mathrm{D}$, et al. Comparative study on risk factors and early outcome of symptomatic distal versus proximal deep vein thrombosis: results from the OPTIMEV study. Thrombosis and haemostasis 2009 Sep; 102(3): 493-500. PubMed PMID: 19718469.

20. Barco S, Corti M, Trinchero A, Picchi C, Ambaglio C, Konstantinides SV, et al. Survival and recurrent venous thromboembolism in patients with first proximal or isolated distal deep vein thrombosis and no pulmonary embolism. Journal of thrombosis and haemostasis JTH 2017 Jul; 15(7): 1436-1442. PubMed PMID: 28439954. Epub 2017/04/26. eng.
21. Galanaud JP, Sevestre MA, Pernod G, Genty C, Richelet S, Kahn SR, et al. Long-term outcomes of cancer-related isolated distal deep vein thrombosis: the OPTIMEV study. Journal of thrombosis and haemostasis JTH. 2017 May;15(5):907-16. PubMed PMID: 28266773. Epub 2017/03/08. eng.

22. Lagerstedt C, Olsson CG, Fagher B, Oqvist B, Albrechtsson $U$. Oral anticoagulants in calf-vein thrombosis. Lancet. 1985 Dec 7;2(8467):1311-2. PubMed PMID: 2866380.

23. Lagerstedt CI, Olsson CG, Fagher BO, Oqvist BW, Albrechtsson U. Need for long-term anticoagulant treatment in symptomatic calf-vein thrombosis. Lancet. 1985 Sep 7;2(8454):515-8. PubMed PMID: 2863541.

24. Schwarz T, Buschmann L, Beyer J, Halbritter K, Rastan A, Schellong S. Therapy of isolated calf muscle vein thrombosis: a randomized, controlled study. J Vasc Surg. 2010 Nov;52(5):1246-50. PubMed PMID: 20630682. Epub 2010/07/16. eng.

25. Righini M, Galanaud JP, Guenneguez H, Brisot D, Diard A, Faisse P, et al. Anticoagulant therapy for symptomatic calf deep vein thrombosis (CACTUS): a randomised, double-blind, placebo-controlled trial. Lancet Haematol. 2016 Dec;3(12):e556-e62. PubMed PMID: 27836513. Epub 2016/11/12. eng.

26. Franco L, Giustozzi M, Agnelli G, Becattini C. Anticoagulation in patients with isolated distal deep vein thrombosis: a meta-analysis. Journal of thrombosis and haemostasis : JTH. 2017 Jun;15(6):1142-54. PubMed PMID: 28316124. Epub 2017/03/21. eng.

27. Pinede L, Ninet J, Duhaut P, Chabaud S, Demolombe-Rague S, Durieu I, et al. Comparison of 3 and 6 months of oral anticoagulant therapy after a first episode of proximal deep vein thrombosis or pulmonary embolism and comparison of 6 and 12 weeks of therapy after isolated calf deep vein thrombosis. Circulation 2001 May 22; 103(20): 2453-2460. PubMed PMID: 11369685.

28. Ferrara F, Meli F, Amato C, Cospite V, Raimondi F, Novo G, et al. Optimal duration of treatment in surgical patients with calf venous thrombosis involving one or more veins. Angiology 2006 AugSep; 57(4): 418-423. PubMed PMID: 17022376.

29. Schulman S, Rhedin AS, Lindmarker P, Carlsson A, Larfars G, Nicol P, et al. A comparison of six weeks with six months of oral anticoagulant therapy after a first episode of venous thromboembolism. Duration of Anticoagulation Trial Study Group. The New England journal of medicine 1995 Jun 22; 332(25): 1661-1665. PubMed PMID: 7760866.

30. Li AY, Woulfe T, Rolfe-Vyson V, Rowland V, Simpson D, Merriman E. Management and outcomes of axial isolated distal deep vein thrombosis at North Shore Hospital, New Zealand: a retrospective audit. Intern Med J 2015 Feb; 45(2): 177-182. PubMed PMID: 25521797. Epub 2014/12/19. eng.

31. . VASA Zeitschrift fur Gefasskrankheiten. 2016 Nov;45 Suppl 95:1-100. PubMed PMID: 27855568. S3-Leitlinie PAVK - Diagnostik, Therapie und Nachsorge der peripheren arteriellen Verschlusskrankheit.

32. Kamphuisen PW, Lee AYY, Meyer G, Bauersachs $\mathrm{R}$, Janas MS, Jarner MF, et al. Clinically relevant bleeding in cancer patients treated for venous thromboembolism from the CATCH study. Jour- 
nal of thrombosis and haemostasis JTH 2018 Mar 24. PubMed PMID: 29573330.

33. Lee AYY, Kamphuisen PW, Meyer G, Bauersachs $\mathrm{R}$, Janas MS, Jarner MF, et al. Tinzaparin vs Warfarin for Treatment of Acute Venous Thromboembolism in Patients With Active Cancer: A Randomized Clinical Trial. Jama 2015 Aug 18; 314(7): 677-686. PubMed PMID: 26284719.

34. Decousus H, Prandoni P, Mismetti P, Bauersachs RM, Boda Z, Brenner B, et al. Fondaparinux for the treatment of superficial-vein thrombosis in the legs. The New England journal of medicine 2010 Sep 23; 363(13): 1222-1232. PubMed PMID: 20860504.

35. Beyer-Westendorf J, Schellong SM, Gerlach $\mathrm{H}$, Rabe E, Weitz JI, Jersemann K, et al. Prevention of thromboembolic complications in patients with superficial-vein thrombosis given rivaroxaban or fondaparinux: the open-label, randomised, noninferiority SURPRISE phase $3 \mathrm{~b}$ trial. The Lancet
Haematology 2017 Mar; 4(3): e105-e13. PubMed PMID: 28219692.

36. Kearon C, Akl EA, Ornelas J, Blaivas A, Jimenez D, Bounameaux H, et al. Antithrombotic Therapy for VTE Disease: CHEST Guideline and Expert Panel Report. Chest 2016 Feb; 149(2): 315-352. PubMed PMID: 26867832 